\title{
The combination of mare's milk and grape polyphenol extract for treatment of dysbiosis induced by dextran sulfate sodium
}

\author{
SAMAT KOZHAKHMETOV ${ }^{1,2.3, \boldsymbol{v}}$, DMITRIY BABENKO ${ }^{4}$, MADIYAR NURGAZIYEV ${ }^{1}$, \\ ALTYNAY TUYAKOVA ${ }^{\mathbf{1}}$, AYAULYM NURGOZHINA ${ }^{\mathbf{1}}$, NURISLAM MUHANBETGANOV ${ }^{\mathbf{1}}$, \\ LAURA CHULENBAYEVA ${ }^{1}$, SHYNGGYS SERGAZY ${ }^{1}$, ALEXANDR GULYAYEV ${ }^{1}$, \\ TIMUR SALIEV ${ }^{5}$, ALMAGUL KUSHUGULOVA ${ }^{1,2,3}$ \\ ${ }^{1}$ Laboratory of Human Microbiome and Longevity, Center for Life Sciences, National Laboratory Astana, Nazarbayev University. Z05H0P9, Kabanbay \\ Batyr avenue 53, block S1, Nur-Sultan, Kazakhstan. Tel.+7 7172 706521, `email: skozhakhmetov@nu.edu.kz \\ ${ }^{2}$ Kazakhstan Society of Researchers of Human Microbiome. Tawelsizdik 34, Z00T2C6, Nur-Sultan, Kazakhstan \\ ${ }^{3}$ SaumalBioTech LLP. Z05H0K9, Kabanbay Batyr avenue 6/4, Nur-Sultan, Kazakhstan \\ ${ }^{4}$ Research Center Karaganda Medical University. M01 K6T3, Gogol street 40, Karagandy, Kazakhstan \\ ${ }^{5}$ S.D. Asfendiyarov Kazakh National Medical University. A05H2A6, Tole Bi Street 94, Almaty, Kazakhstan
}

Manuscript received: 12 April 2020. Revision accepted: 28 April 2020.

\begin{abstract}
Kozhakhmetov S, Babenko D, Nurgaziyev M, Tuyakova A, Nurgozhina A, Muhanbetganov N, Chulenbayeva L, Sergazy S, Gulyayev A, Saliev T, Kushugulova A. 2020. The combination of mare's milk and grape polyphenol extract for treatment of dysbiosis induced by dextran sulfate sodium. Biodiversitas 21: 2275-2280. This study showed the potential of a biological product based on mare's milk and a complex of grape polyphenols to modulate intestinal microflora after dextran sulfate sodium (DSS)-induced dysbiosis. Rat ulcerative colitis has been developed using intra-gastric administration of $10 \%$ DSS solution. To track changes in the structure of the microbiome at all stages of the study, the next-generation sequencing of the $16 \mathrm{~S}$ rRNA gene section and LotuS conveyor were used. The results of sequencing demonstrated a decrease in biological diversity of microbiota after the induction of colitis, and recovery after 7 days of use of the (MMGPE). The product induced the structural changes of the microbiome damaged by DSS. Representatives of SCFA producing bacteria increased concentrations of Prevotella, Alloprevotella, Lactobacillus, Ruminococcaceae, and Blautia.
\end{abstract}

Keywords: DSS-induced dysbiosis, gut microbiome, microbial diversity, MMGPE, rats

\section{INTRODUCTION}

The microflora of the human intestine is a complex structured ecosystem, which includes parietal intestinal flora and transit microorganisms from food. Intestinal microorganisms are a dynamic system that depends on many factors such as food, environment, pre-existing diseases, etc. Destruction of the microbial ecosystem structure is of great importance in the pathogenesis of various diseases, for example, for the development of inflammatory bowel syndrome, Crohn's disease, and ulcerative colitis (UC). In fact, the patients with ulcerative colitis suffer from the impaired diversity and stability of the intestinal microbiome with a decrease in Firmicutes and an increase in the number of Bacteroidetes and facultative anaerobes (Shen et al. 2018). As a result of ulcerations, the intestinal wall ceases to be a normal niche for the living of microorganisms and performs a barrier function. This, in turn, can impact digestive processes in the intestine, leading to a decrease in the mucus layer, a disruption in the shape of epithelial cells, and dystrophic phenomena in the epithelium (Pei et al. 2019). UC therapy today is not satisfactory, and it does not lead to complete remission. In addition, patients often have relapses, because standard treatment is usually not effective enough for a stable remission.

To date, drugs based on 5-aminosalicylic acid are used as a classic approach for UC therapy. However, there are attempts to employ various probiotics, but the benefits of their use in comparison with placebo have not been identified yet (Derwa et al. 2017). Unlike probiotics, fecal microbial transplantation leads to persistent remission of ulcerative colitis (Moayyedi et al. 2015; Costello et al. 2019; Tian et al. 2019). Another promising direction is the use of products that stimulate the growth of certain microflora, such as polyphenols and mare's milk. Numerous studies have shown how polyphenols can contribute to changes in the microbial composition of the intestine, at the same time there are no reports on their effect on the treatment of UC. It was found out that resveratrol extracted from grapes had anti-inflammatory activity along with stimulation of the growth of Lactobacillus and Bifidobacterium after DSS-induced colitis ( $\mathrm{Hu}$ et al. 2019). Similarly, Chen et al. demonstrated that treatment with resveratrol resulted in an increase of concentrations of Bacteroides and Akkermansia (Chen et al. 2016). Another study showed that Akkermansia produces a significant amount of SCFA when polyphenols are added (Naito et al. 2018). Mare's milk, also being a prebiotic, stimulates the growth of certain bacteria. In addition, mare's milk has a complex of biologically active compounds including vitamins: $\mathrm{A}, \mathrm{B}_{1}, \mathrm{~B}_{2}, \mathrm{~B}_{6}, \mathrm{~B}_{9}, \mathrm{~B}_{12}, \mathrm{C}$, trace elements $\mathrm{Na}, \mathrm{Ca}, \mathrm{K}, \mathrm{P}, \mathrm{Fe}$, $\mathrm{Mg}, \mathrm{Cu}, \mathrm{I}, \mathrm{S}, \mathrm{Si} . \mathrm{Zn}, \mathrm{Co}$, lysozyme, lactoferrin. In our study, we studied the therapeutic potential of a biologically active substance based on mare's milk and a polyphenol complex against ulcerative colitis (Rather et al. 2020). 


\section{MATERIALS AND METHODS}

\section{Ethical approval}

The study was approved by the local ethics committee of the Center for Life Sciences, National Laboratory of Astana, Nazarbayev University, Nur-Sultan, Kazakhstan (approval No. 20 dated 22 September 2017).

\section{Animals study}

The study was carried out on 15 laboratory animals (male Wistar rats with an average body weight range of 250-280) in the vivarium of National Center for Biotechnology (Nur-Sultan, Kazakhstan) with a standard ration and care. Rats were placed in separate cages in a room free of pathogens and for acclimatization 7 days before the start of the experiment. During acclimatization and experiment, rats consumed a standard commercially available chow. The animals were kept and the experiments conducted in compliance with the Guide for the Care and Use of Laboratory Animals (National Research Council 2011) and the ethical principles of the European Convention for the Protection of Vertebrate Animals used for Experimental and Other Scientific Purposes (Council of Europe 2006).

Colitis in male rats was reproduced using a 10\% DSS solution (Dextran sodium sulfate, MW-40 kDa, Sigma Aldrich) (Chassaing et al. 2014), which was administered to the rats intragastrically using a probe for 7 days in a volume of $5 \mathrm{ml}$. A $10 \%$ DSS solution was prepared ex tempore before use. Intact rats received an equivolume amount of drinking water intragastrically using a probe (manipulation control). The studied product (MMGPE) to study the efficacy against microbial dysbiosis in the DSSinduced colitis model in rats was administered intragastrically at a dose of $500 \mathrm{mg} / \mathrm{kg}$ of animal body weight, once a day for 7 days after the end of a 7-day course of taking a $10 \%$ solution DSS. Three months old Wistar rats with an average body weight range of 250-280 $\mathrm{g}$ were randomly divided into 3 groups: (i) $\mathrm{HC}$ : healthy animals (without colitis) received drinking water intragastrically instead of 10\% DSS for 7 days and then another 7 days instead of treatment $(n=5)$. (ii) $E G$ : experimental group animals (with colitis) received a $10 \%$ dextran sulfate sodium (DSS) solution for 7 days, and study product intragastrically at a dose of $500 \mathrm{mg} / \mathrm{kg}$ body weight once per day for 7 days $(n=5)$. (iii) CG: comparison group animals (with colitis) received a 10\% DSS solution for 7 days and as a treatment the 5-ASA (5-aminosalicylic acid) intragastrically at a dose of $100 \mathrm{mg} / \mathrm{kg}$ of animal body weight once for 7 days $(n=5)$. Rats were removed from the experiment by an overdose of carbon dioxide (Hewett et al. 1993). Fecal samples were collected before and after the experiment and tested for consistency and color. Other tested parameters including intestinal permeability and body weight.

\section{Measurement of the rats gut microbiota}

DNAs were isolated from fecal using the QIAamp DNA Mini Kit (Qiagen, 51306). The concentration of double-stranded DNA in isolated samples was determined using a Qubit 2.0 instrument and a Qubit dsDNA HS Assay kit (ThermoFisher, catalog number 32853).

Library for Next-generation sequencing (NGS) generated with NEXTflex ${ }^{\circledR}$ 16S V1-V3 Amplicon-Seq Kit (PerkinElmer, catalog number NOVA-4202-04), according to the manufacturer's instructions. The library quality was quantified by Qubit dsDNA HS Assay Kit with the Qubit 2.0 fluorometer system (Invitrogen, Life Technologies, Grand Island, NY, USA). Amplicons were sequenced on the MiSeq instrument (Illumina Systems).

Demultiplexing, filtering, denoise, chimeric sequences, and determining OTU and taxonomic identification were performed using LotuS pipeline (Hildebrand et al. 2014).

Analysis of alpha diversity to assess the abundance of the community, the calculation of alpha biodiversity (Shannon indices), beta biodiversity as well as the construction of taxonomic distribution at the phylum and genus level were performed using vegan (Oksanen et al. 2019) and phyloseq R packages (v.1.24.2) (McMurdie and Holmes 2013) and graphs were generated using web-based platform for comprehensive analysis - MicrobiomeAnalyst (Chong et al. 2020).

\section{Statistical analysis}

Non-parametric Mann-Whitney (MW) and KruskalWallis (KW) tests were used for comparing two or more groups, respectively. The raw read counts were normalized by the total number of reads. A metagenomic biomarker discovery approach, Linear discriminant analysis Effect Size (LEfSe), was used to identify the microbial components whose sequences were statistically different between groups. For LEfSe, Kruskal-Wallis and pairwise Wilcoxon tests were performed, followed by Linear discriminant analysis (LDA) to assess the effect size of each differentially abundant taxon. Bacteria with markedly increased numbers were defined as those with an LDA score $(\log 10)$ of over 2.

\section{RESULTS AND DISCUSSION}

In this study, a biological product based on grape polyphenols (Kazakhstan breeding Cabernet Sauvignon) and mare's milk was tested for the ability to restore the intestinal microflora of rats after DSS-induced colitis. The results showed that the dynamics of body weight of the EG group did not change compared to HC group animals.

Disease Activity Index (DAI) was calculated on a scale of 0 to 4 ; weight loss (0: no; $1: 0-5 \% ; 2: 5-10 \% ; 3: 10-$ $20 \%$; 4, > 20\%); stool consistency ( 0 : normal; 2 : loose stools; 4: watery diarrhea); bleeding (0: no; 1 : traces; 2 : weak hidden blood; 3: obvious hidden blood; 4: severe bleeding). After 1 week of taking DSS, the stool was mainly soft, and in some cases pasty, while the HC group animals have stools. No bleeding was observed. DAI showed no difference from CG taking 5-aminosalicylic acid. To determine the ability of the developed product to modulate the microflora, rats stool samples were collected before treatment and after 7 days of drug administration. In total, 30 stool specimens were collected from 15 male rats. 
Rat feces samples were collected in sterile centrifuge tubes and immediately frozen at $-80^{\circ} \mathrm{C}$.

High throughput sequencing yielded an average of 72,541 read operations per sample. Analysis of the gut rat microbiome revealed a high taxonomic diversity of bacteria. LotuS based microbial community analysis yielded a total of 3341 operational taxonomic units (OTUs). Based on phylogenetic analysis using the $16 \mathrm{~S}$ rRNA gene SILVA database more reads were classified on level of phylum as Bacteroidetes, Firmicutes, Proteobacteria, Actinobacteria, Tenericutes, Spirochaetes, Candidatus saccharibacteria (Figure 1).
To determine the diversity of intestinal microbial communities in the various studied groups, the $\alpha$-diversity metric implemented in $\mathrm{R}$. was used. The most representative bacterial genera in the group before using the biological product were: Lactobacillus, Prevotella, Romboutsia, Helicobacter, Bacteroides, Ruminococcus, Treponema Butyricicoccus, Parasutterella. However, the use of $10 \%$ DSS led to a decrease in biodiversity and $\alpha$ diversity in the EG group (Figure 2.A), whilst the Shanon index was increased in the group taking 5-ASA (5aminosalicylic acid) (Figure 2.B), and in the control group it remained at the same level (Figure 2.C).

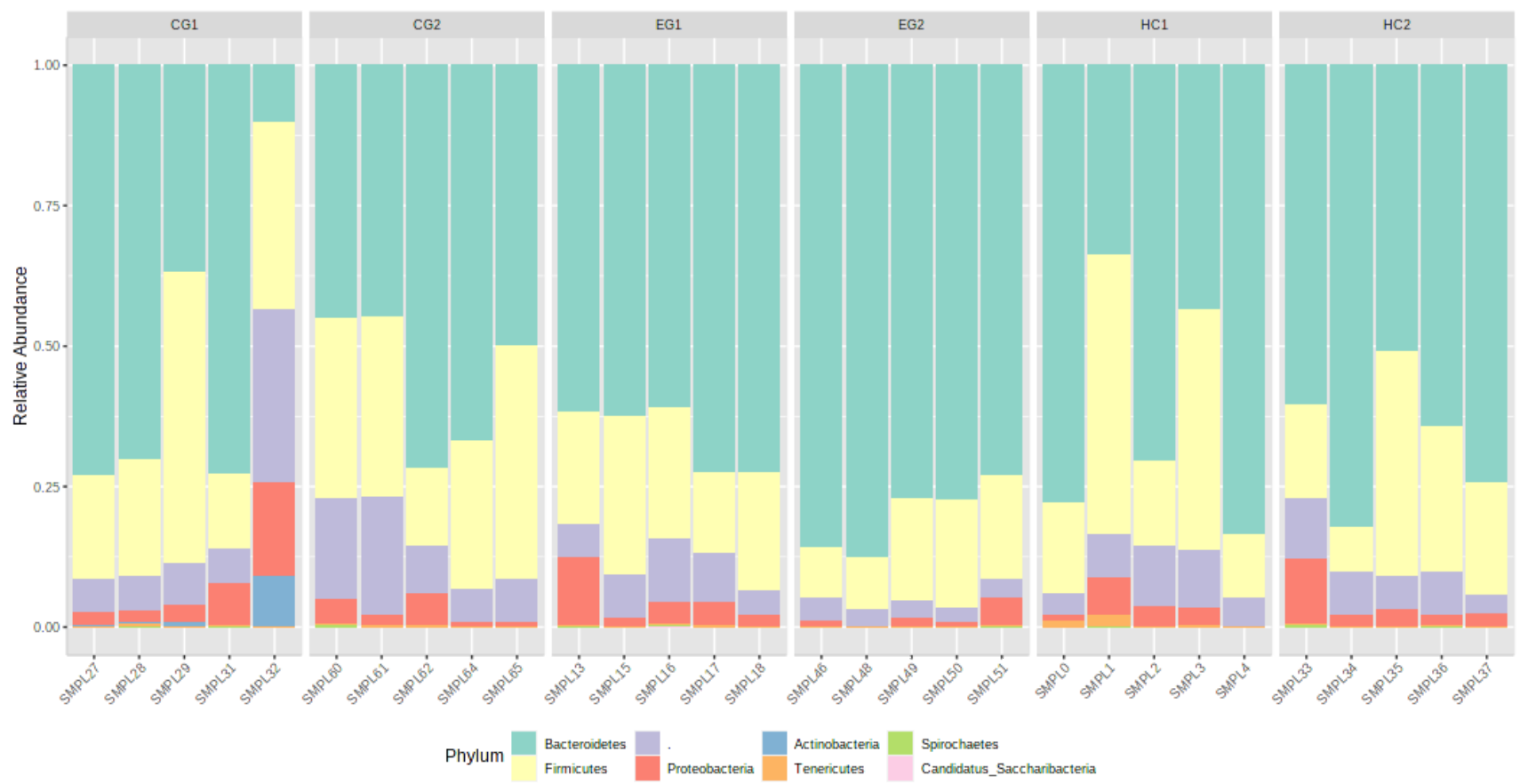

Figure 1. Bacterial Phylum abundance in rat's fecal before and after 7 days of treatment CG1, EG1 HC1-group animals before treatment; CG2, EG2, HC2-group animals after treatment

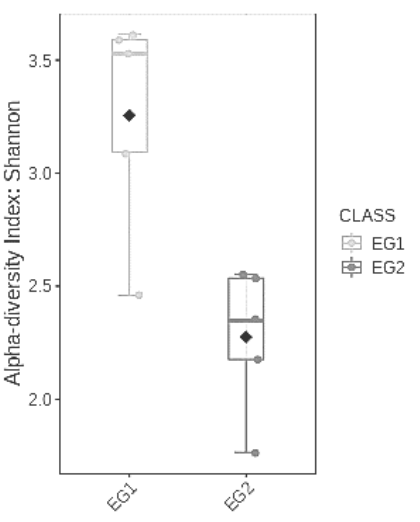

A

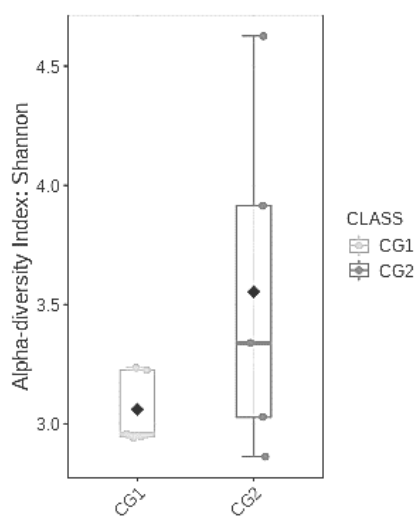

B

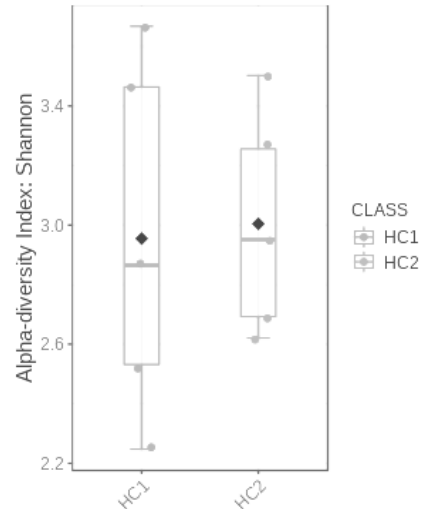

C

Figure 2. $\alpha$-diversity fecal bacteria in EG, HC and CG $(\mathrm{p}<0.05)$ 


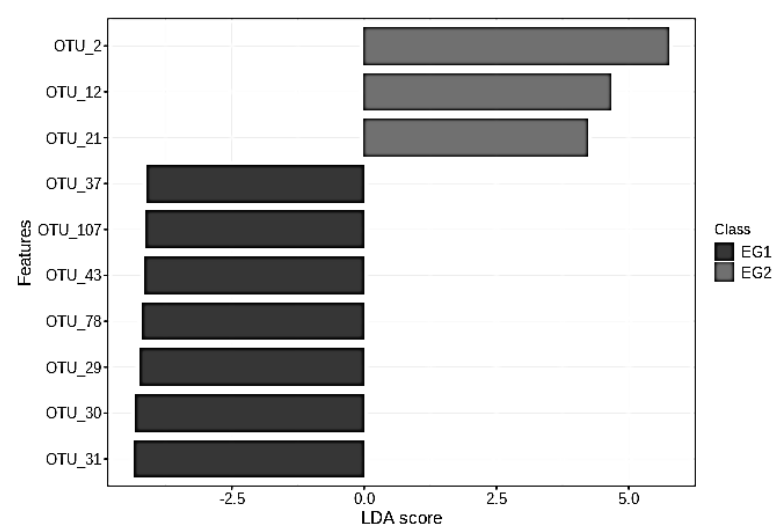

A

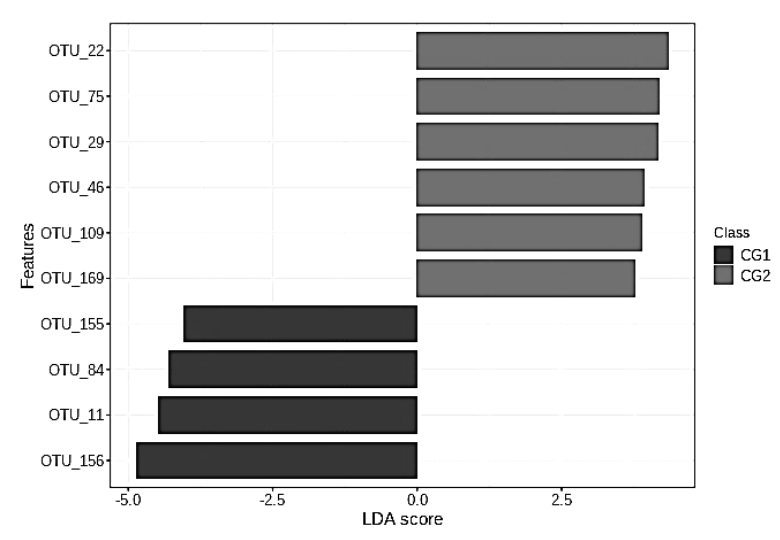

B

Figure 3. Linear Discriminant Analysis (LDA) Effect Size (LEfSe) OTU level, p-value $<0.05$

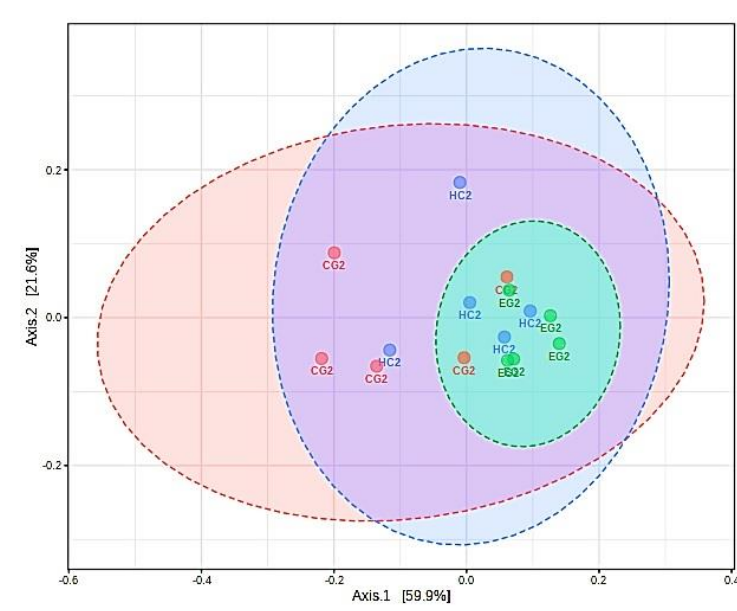

A
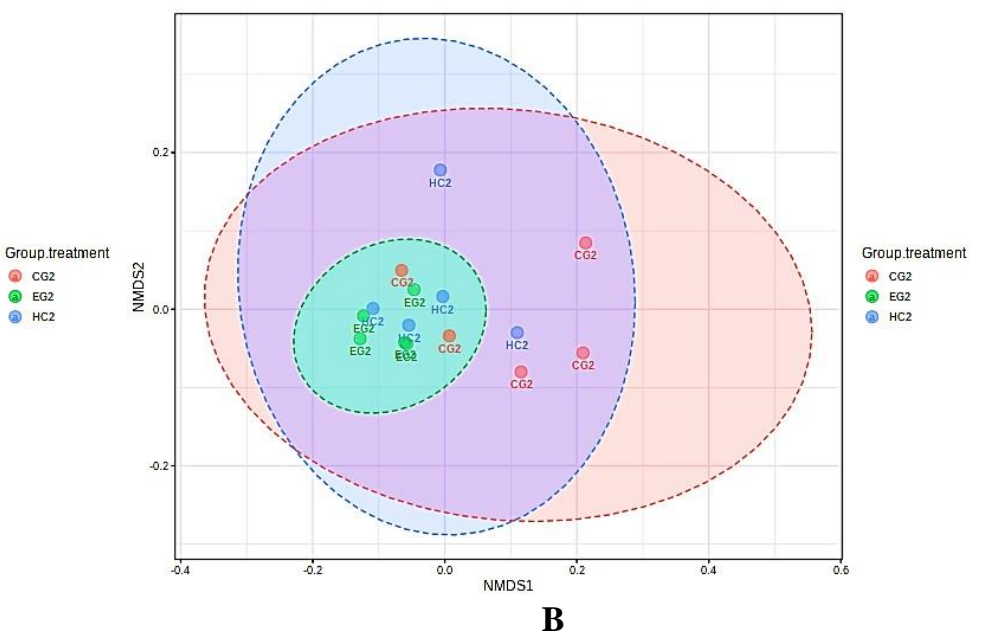

Figure 4. Effect of administration DSS on rat's intestinal microbiome. A. PCoA analyze [PERMANOVA] F-value: 5.8788; R-squared: 0.42358; p-value < 0.019. B. NMDS analyze [PERMANOVA] F-value: 3.2954; R-squared: 0.35452; p-value < 0.011

LDA in EG revealed an increase in the genus Prevotella (OTU_2) (Figure 3.A), Bacteroidales (OTU_12), and genus Lactobacillus (OTU_21) after treatment with a biological product. While family Lachnospiraceae (OTU_37, OTU_107), genus Helicobacter (OTU_43), unknown bacteria of phylum Bacteroidetes (OTU_29, OTU_30, OTU_31, OTU_78) decreased. In comparison group CG after treatment with 5-ASA led to an increase in order Clostridiales (OTU_22), Alphaproteobacteria (OTU_169), and decreased Gammaproteobacteria (OTU_11), Actinobacteria genus Rothia (OTU_156) (Figure 3.B). In addition, Differential Abundance Analysis Methods (DeSeq2) in the group receiving the biological product revealed an increase in the genera Prevotella and Alloprevotella.

The decrease in the biodiversity of the intestinal bacterial flora can be explained by ulcerations (induced by DSS) and local activation of neutrophils and macrophages. PCoA and NMDS analyzes showed the difference between microbial communities after treatment by CG2 with 5- aminosalicylic acid, EG2 biological product based on mare's milk and grape polyphenol concentrate (Figure 4). The data obtained demonstrated an increase in the growth of bacteria Ruminococcaceae (OTU_174) and Bacteroidales (OTU_223).

In the group received the (MMGPE), a decrease in the genera Parabacteroides (OTU_295) and Turicibacter (OTU_118) (Figure 2) and an increase in proteolytic and Proteobacteria including from family Moraxellaceae were observed.

\section{Discussion}

Ulcerative colitis is a chronic disease of the intestinal mucosa that requires long-term medication and regular check-ups. The disease is often accompanied by microbial dysbiosis (Xie et al. 2019). Microbial dysbiosis in UC is characterized by an increase of bacteria Proteobacteria and Bacteroidetes and a decrease of bacteria Firmicutes phylum and Euryarchaeota phylum. In addition, a decrease in the index of alpha diversity, the relative representation of 
bacteria producing butyrate, hydrogen; increase in relative representation of Ruminococcus was also reported (Danilova et al. 2019).

In this study, in the healthy group of animals, the top 10 taxa included the following bacterial genera: Lactobacillus, Prevotella, Romboutsia, Helicobacter, Mycoplasma, Rikenella, Parabacteroides, Bacteroides, Parasutterella, Ruminococcus. After the formation of the DSS-induced colitis in top 10 taxa model, Treponema, Butyricicoccus, Turicibacter was introduced. At the same time, the number of Mycoplasma, Rikenella, and Parabacteroides decreased. The results are consistent with published data (Gao et al. 2018).

Prebiotics are known for their ability to selectively stimulate the activity of intestinal bacteria strains associated with health such as Bifidobacteria and Lactobacilli, which are considered beneficial to human health. In addition, prebiotics can enhance the guts barrier function and host immunity, SCFA production, and suppress the populations of potentially pathogenic bacteria (Slavin 2013). We demonstrated that the use of a (MMGPE) of Kazakhstan selection led to a shift in the balance of intestinal microflora. These findings can be potentially employed for the treatment of ulcerative colitis. The genus included in the top 10 taxa: Lactobacillus, Prevotella, Romboutsia, Helicobacter, Rikenella, Bacteroides, Parasutterella, Ruminococcus, Treponema, and Blautia.

Particularly, we detected an increase in the representatives of Ruminococcaceae family (OTU_174), which are mucolytic bacteria (Ruminococcus gnavus and Ruminococcus torques). These strains are the main components of mucus-mucins of the intestinal mucosa, and they play a critical role in gut permeability (Hall et al. 2017). In our work, we showed an increase in the growth of Ruminococcaceae (Figure 2.A) caused by ulcerative colitis (induced by the administration of $10 \%$ DSS). The obtained results once again demonstrated the correlation between an increase in the group of mucolytic bacteria and signs of inflammatory bowel diseases (Png et al. 2010). The results indicate that an increase in mucosa-associated bacteria is associated with ulcerations and a decrease in the host resistance. The detected increase of Bacteroidales (Figure 1.B) in the EG group can be a result of antimicrobial effect of grape polyphenols. In fact, polyphenols can inhibit IBD and modulate the intestinal microflora (Snopek et al. 2018). In our study, we observed low biodiversity after the application of the (MMGPE) Shannon index 2.5 (Figure 1.A) in comparison with the group that was treated with 5ASA, Shannon index 3.5 (Figure 1.B).

LefSe linear discriminant analysis (LDA) was used to determine significant differences in groups by genus ( $\mathrm{p}$ <0.05). The LEfSe taxonomic cladogram (Figure 3) showed critical bacterial changes. At the generic level, an increase in the relative abundance of Prevotella (OTU_2), unknown order Bacteroidales (OTU_12) and Lactobacillus (OTU_21) were revealed. At the same time, the number of populations Lachnospiraceae (OTU_37, OTU_107), Helicobacter (OTU_43) was decreased. In addition, a high frequency of bacteria of the genus Alloprevotella (producers of SCFA) was detected by using DeSeq2 technique. The increased number of Prevotellaceae in the microbiota of rats treated by a biological product can be explained by the activity of polyphenol component (Etxeberria et al. 2015). The detected increase of Lactobacillus was due to the stimulating effect of mare's milk (Fotschki et al. 2016).

In fact, the microbiota's shift can directly affect the vital functions of the organism (Järbrink-Sehgal and Andreasson 2020; Wan et al. 2020; Wang et al. 2020). In this study, we observed an increase in the strains producing SCFA, including Prevotella, Alloprevotella, Lactobacillus, Ruminococcaceae, Blautia as a result of the application of the (MMGPE). Moreover, a decrease in the number of Helicobacter and other representatives of pathogenic gut flora was also detected. Our findings suggest that the natural biologically active (MMGPE) possess therapeutic potential for the treatment of ulcerative colitis. However, more studies are required in order to validate its efficacy and clinical relevance.

\section{ACKNOWLEDGEMENTS}

The authors are thankful to the Ministry of Education and Science Republic of Kazakhstan for funding this research work (grants: AP05134659, AP05135073, BR05236508)

\section{REFERENCES}

Chassaing B, Aitken JD, Malleshappa M, Vijay-Kumar M. 2014. Dextran sulfate sodium (DSS)-induced colitis in mice. Curr Protoc Immunol 104 (1): 15-25.

Chen M, Yi L, Zhang Y, Zhou X, Ran L, Yang J, Zhu J, Zhang Q, MiM. 2016. Resveratrol Attenuates Trimethylamine-N-Oxide (TMAO)Induced Atherosclerosis by Regulating TMAO Synthesis and Bile Acid Metabolism via Remodeling of the Gut Microbiota. MBio 7: e02210-e2215. DOI: 10.1128/mBio.02210-15

Chong J, Liu P, Zhou G, Xia J. 2020. Using MicrobiomeAnalyst for comprehensive statistical, functional, and meta-analysis of microbiome data. Nat Protoc 150:799-821. DOI: 10.1038/s41596019-0264-1

Council of Europe. 2006. Appendix A of the European Convention for the Protection of Vertebrate Animals used for Experimental and Other Scientific Purposes Accommodation and Care of Animals (Article 5 of the Convention) Approved by Multilateral Consultation Cons 123 (2006) 3. Strasburg: Council of Europe.

Costello SP, Hughes PA, Waters O, Bryant RV, Vincent AD, Blatchford P, Katsikeros R, Makanyanga J, Campaniello MA, Mavrangelos C, Rosewarne CP, Bickley C, Peters C, Schoeman MN, Conlon MA, Roberts-Thomson IC, Andrews JM. 2019. Effect of fecal microbiota transplantation on 8-week remission in patients with ulcerative colitis: a randomized clinical trial. JAMA 321: 156-164. DOI: 10.1001/jama.2018.20046

Danilova N, Abdulkhakov S, Grigoryeva T, Markelova M, Vasilyev I, Boulygina E, Ardatskaya M, Pavlenko A, Tyakht A, Odintsova A, Abdulkhakov R. 2019. Markers of dysbiosis in patients with ulcerative colitis and Crohn's disease. Ter Arkh 91: 17-24. DOI: 10.26442/00403660.2019.04.000211

Derwa Y, Gracie DJ, Hamlin PJ, Ford AC. 2017. Systematic review with meta-analysis: the efficacy of probiotics in inflammatory bowel disease. Aliment Pharmacol Ther 46: 389-400. DOI: 10.1111/apt.14203

Etxeberria U, Arias N, Boqué N, Macarulla MT, Portillo MP, Martínez JA, Milagro FI. 2015. Reshaping faecal gut microbiota composition 
by the intake of trans-resveratrol and quercetin in high-fat sucrose diet-fed rats. J Nutr Biochem 26: 651-660. DOI 10.1016/j.jnutbio.2015.01.002

Fotschki J, Szyc AM, Laparra JM, Markiewicz LH, Wróblewska B. 2016 Immune-modulating properties of horse milk administered to mice sensitized to cow milk. J Dairy Sci 99: 9395-9404. DOI: 10.3168/jds.2016-11499

Gao Z, Chen K-Y, Mueller O, Zhang H, Rakhilin N, Chen J, Shen X. 2018. Microbiota of Inflammatory Bowel Disease Models. 0th Int Eng Med Biol Conf, Honolulu, Hawaii, July 17-21, 2018. 2374-2377. DOI: $10.1109 /$ EMBC.2018.8512848

Hall AB, Yassour M, Sauk J, Garner A, Jiang X, Arthur T, Lagoudas GK, Vatanen T, Fornelos N, Wilson R, Bertha M, Cohen M, Garber J, Khalili H, Gevers D, Ananthakrishnan AN, Kugathasan S, Lander ES, Blainey P, Vlamakis H, Xavier RJ, Huttenhower C. 2017. A novel Ruminococcus gnavus clade enriched in inflammatory bowel disease patients. Genome Med 9: 103. DOI: 10.1186/s13073-017-0490-5

Hewett TA, Kovacs MS, Artwohl JE, Bennett BT. 1993. A comparison of euthanasia methods in rats, using carbon dioxide in prefilled and fixed flow rate filled chambers. Lab Anim Sci 43: 579-582.

Hildebrand F, Tadeo R, Voigt AY, Bork P, Raes J. 2014. LotuS: an efficient and user-friendly OTU processing pipeline. Microbiome 2: 30-37. DOI: 10.1186/2049-2618-2-30

Hu Y, Chen D, Zheng P, Yu J, He J, Mao X, Yu B. 2019. The Bidirectional Interactions between Resveratrol and Gut Microbiota: An Insight into Oxidative Stress and Inflammatory Bowel Disease Therapy. Biomed Res Int 5403761. DOI: 10.1155/2019/5403761

Järbrink-Sehgal E, Andreasson A. 2020. The gut microbiota and mental health in adults. Curr Opin Neurobiol 62: 102-114. DOI: 10.1016/j.conb.2020.01.016

McMurdie PJ, Holmes S. 2013. phyloseq: an R package for reproducible interactive analysis and graphics of microbiome census data. PLoS One 8: e61217. DOI: 10.1371/journal.pone.0061217

Moayyedi P, Surette MG, Kim PT, Libertucci J, Wolfe M, Onischi C, Armstrong D, Marshall JK, Kassam Z, Reinisch W, Lee CH. 2015 Fecal Microbiota Transplantation Induces Remission in Patients With Active Ulcerative Colitis in a Randomized Controlled Trial Gastroenterology 149: 102-109. DOI: 10.1053/j.gastro.2015.04.001

Naito Y, Uchiyama K, Takagi T. 2018. A next-generation beneficial microbe: Akkermansia muciniphila. J Clin Biochem Nutr 63: 33-35. DOI: $10.3164 /$ jcbn. $18-57$

National Research Council. 2011. Guide for the Care and Use of Laboratory Animals: Eighth Edition. Washington, DC: The National Academies Press. DOI: 10.17226/12910.
Oksanen J, Blanchet FG, Friendly M, Kindt R, Legendre P, Mcglinn D. 2019. Package "vegan" Title Community Ecology Package. Commun Ecol Packag 2 (9): 1-297.

Pei L, Ke Y, Zhao H, Wang L, Jia C, Liu W, Fu Q, Shi M, Cui J, Li S. 2019. Role of colonic microbiota in the pathogenesis of ulcerative colitis. BMC Gastroenterol 19: 10. DOI: 10.1186/s12876-019-0930-3

Png CW, Lindén SK, Gilshenan KS, Zoetendal EG, McSweeney CS, Sly LI, McGuckin MA, Florin THJ. 2010. Mucolytic bacteria with increased prevalence in IBD mucosa augment in vitro utilization of mucin by other bacteria. Am J Gastroenterol 105: 2420-2428. DOI: 10.1038/ajg.2010.281

Rather IA, Bajpai VK, Ching LL, Majumder R, Nam G-J, Indugu N, Singh P, Kumar S, Hajrah NH, Sabir JSM, Kamli MR, Park Y-H. 2020. Effect of a bioactive product SEL001 from Lactobacillus sakei probio65 on gut microbiota and its anti-colitis effects in a TNBSinduced colitis mouse model. Saudi J Biol Sci 27: 261-270. DOI: DOI: 10.1016/j.sjbs.2019.09.004

Shen Z-H, Zhu C-X, Quan Y-S, Yang Z-Y, Wu S, Luo W-W, Tan B, Wang X-Y. 2018. Relationship between intestinal microbiota and ulcerative colitis: Mechanisms and clinical application of probiotics and fecal microbiota transplantation. World J Gastroenterol 24: 5-14. DOI: $10.3748 /$ wjg.v24.i1.5

Slavin J. 2013. Fiber and prebiotics: mechanisms and health benefits. Nutrients 5: 1417-1435. DOI: 10.3390/nu5041417

Snopek L, Mlcek J, Sochorova L, Baron M, Hlavacova I, Jurikova T, Kizek R, Sedlackova E, Sochor J. 2018. Contribution of red wine consumption to human health protection. Molecules 23: 1684. DOI: 10.3390/molecules23071684

Tian Y, Zhou Y, Huang S, Li J, Zhao K, Li X, Wen X., Li X. 2019. Fecal microbiota transplantation for ulcerative colitis: a prospective clinical study. BMC Gastroenterol 19: 116. DOI: 10.1186/s12876-019-1010-4

Wan MLY, Co VA, El-Nezami H. 2020. Dietary polyphenol impact on gut health and microbiota. Crit Rev Food Sci Nutr. DOI: 10.1080/10408398.2020.1744512

Wang J, Xiong K, Zhao S, Zhang C, Zhang J, Xu L, Ma A. 2020. Longterm effects of multi-drug-resistant tuberculosis treatment on gut microbiota and its health consequences. Front Microbiol 11: 53.

Xie J, Liu Y, Chen B, Zhang G, Ou S, Luo J, Peng X. 2019. Ganoderma lucidum polysaccharide improves rat DSS-induced colitis by altering cecal microbiota and gene expression of colonic epithelial cells. Food Nutr Res 63: 1559. DOI: 10.29219/fnr.v63.1559. 